\title{
Evaluation of the chicken transcriptome by SAGE of $B$ cells and the DT40 cell line
}

\author{
Matthias B Wahl ${ }^{\dagger 1,4}$, Randolph B Caldwell ${ }^{\dagger 1}$, Andrzej M Kierzek ${ }^{2,4,5}$, \\ Hiroshi Arakawa1, Eduardo Eyras ${ }^{3}$, Nina Hubner ${ }^{1}$, Christian Jung1, \\ Manuel Soeldenwagner ${ }^{1}$, Manuela Cervelli ${ }^{1}$, Yan-Dong Wang1, \\ Volkmar Liebscher ${ }^{3}$ and Jean-Marie Buerstedde*1
}

\begin{abstract}
Address: ${ }^{1}$ Institute of Molecular Radiobiology, GSF, Ingolstädter Landstr. 1, D-85764 Neuherberg, Germany, ${ }^{2}$ Laboratory of Systems Biology Institute of Biochemistry and Biophysics, Polish Academy of Sciences, Pawinskiego 5a, 02-106 Warszawa, Poland, ${ }^{3}$ Research Group of Biomedical Informatics, IMIM/Universidad Pompeu Fabra/Centre de Regulacio Genomica, E08003 Barcelona, Spain, ${ }^{4}$ Institute of Biomathematics, GSF, Ingolstädter Landstr. 1, D-85764 Neuherberg, Germany and ${ }^{5}$ Stowers Institute for Medical Research, 1000 E. 50th Street, Kansas City, MO 64110, USA

Email: Matthias B Wahl - mbw@stowers-institute.org; Randolph B Caldwell - randolph.caldwell@gsf.de; Andrzej M Kierzek - andrzejk@ibb.waw.pl; Hiroshi Arakawa - hiroshi@gsf.de; Eduardo Eyras - eeyras@imim.es;

Nina Hubner - nina.hubner@web.de; Christian Jung - j-christian@t-online.de; Manuel Soeldenwagner - soeldi@t-online.de; Manuela Cervelli - cervelli@uniroma3.it; Yan-Dong Wang - Yan.wang@gsf.de; Volkmar Liebscher - liebscher@gsf.de; Jean-

Marie Buerstedde* - buerstedde@gsf.de

* Corresponding author †Equal contributors
\end{abstract}

Published: 21 December 2004

BMC Genomics 2004, 5:98 doi:10.1 I86/147|-2164-5-98

This article is available from: http://www.biomedcentral.com/I47I-2/64/5/98

(c) 2004 Wahl et al; licensee BioMed Central Ltd.

This is an Open Access article distributed under the terms of the Creative Commons Attribution License (http://creativecommons.org/licenses/by/2.0), which permits unrestricted use, distribution, and reproduction in any medium, provided the original work is properly cited.

\begin{abstract}
Background: The understanding of whole genome sequences in higher eukaryotes depends to a large degree on the reliable definition of transcription units including exon/intron structures, translated open reading frames (ORFs) and flanking untranslated regions. The best currently available chicken transcript catalog is the Ensembl build based on the mappings of a relatively small number of full length cDNAs and ESTs to the genome as well as genome sequence derived in silico gene predictions.
\end{abstract}

Results: We use Long Serial Analysis of Gene Expression (LongSAGE) in bursal lymphocytes and the DT40 cell line to verify the quality and completeness of the annotated transcripts. $53.6 \%$ of the more than 38,000 unique SAGE tags (unitags) match to full length bursal cDNAs, the Ensembl transcript build or the genome sequence. The majority of all matching unitags show single matches to the genome, but no matches to the genome derived Ensembl transcript build. Nevertheless, most of these tags map close to the 3' boundaries of annotated Ensembl transcripts.

Conclusions: These results suggests that rather few genes are missing in the current Ensembl chicken transcript build, but that the $3^{\prime}$ ends of many transcripts may not have been accurately predicted. The tags with no match in the transcript sequences can now be used to improve gene predictions, pinpoint the genomic location of entirely missed transcripts and optimize the accuracy of gene finder software. 


\section{Background}

The definition of transcription units within a finished genome sequence in higher eukaryotes is challenging and relies on genome mapping of cDNAs and ESTs backed up by theoretical gene finder algorithms. An increasing number of gene sequences from model organisms have made the prediction of well conserved ORFs easier, but less conserved coding and untranslated regions are difficult to detect. The best way to unambiguously define transcription units is full length cDNAs, but large scale projects are expensive in terms of labor and costs. One also needs to bear in mind that some cDNAs elude detection, because unusual secondary structure or toxicity inhibits reverse transcription or cloning.

SAGE investigates the transcription profile of a given cell sample by large scale sequencing of short cDNA tags derived from the bulk mRNA [1,2]. Whereas tag mapping to the cDNA and genome databases of the organism indicates the type of expressed genes, the prevalence of individual tags within the library reflects their relative levels of expression. Since SAGE tags are only short sequences, they can be collected more easily in higher numbers than ESTs and full length cDNA sequences. The potential of SAGE to discover new or better define already known transcription units is particularly advantageous in situations where the entire genome sequence of an organism has been determined, but gene predictions based on theoretical algorithms and the mapping of a relatively small number of EST and CDNA sequences remain tentative. LongSAGE generates longer tags of 21 bases as compared to the classical SAGE protocol and is therefore better suited for the unambiguous assignments of tag to genome sequences $[3,4]$.

Cellular and molecular features of early B cell development [5] and lymphoma formation $[6,7]$ have been extensively studied in the chicken. Gene expression signatures of primary bursal B cells, pre-neoplastic and neoplastic lymphoma cells were collected by microarray hybridizations in a first attempt to identify genes up- or down-regulated during myc-induced B cell lymphoma development [8].

The whole chicken genome including a genome scale transcript build from Ensembl [9] and a collection of bur- sal full-length cDNAs [10] have recently been released. We describe here the mapping of large collections of SAGE tags from bursal lymphocytes and DT40 to these reference datasets to evaluate the quality of the transcript build. Furthermore, the transcription profiles of bursal cells and DT40 as defined by this first SAGE analysis in the chicken should lead to a better understanding of B cell transformation and facilitate the selection of candidate genes for disruption in DT40 [11].

\section{Results and Discussion Generation of SAGE tag libraries and SAGE tags collections}

Two SAGE libraries, named busage and dt40sage, were made from the bursa of Fabricius and DT40 cells using the LongSAGE technique which generates tags of 21 nucleotides in length and therefore decreases the likelihood of ambiguous matches [3,4]. Of the 129,568 tags collected, about equal numbers were derived from the busage and the $\mathrm{dt}$ 40sage libraries respectively (Table 1 ). In total 38,212 unitags were derived from the SAGE tags of both libraries. The library from bursal cells and the DT40 cell line seem to be similar with regard to the number of extracted unitags and the average counts of matching SAGE tags. Underlying a standard binomial model, one would expect to find a special Unitag among the busage tags or $\mathrm{dt} 40$ tags with a probability of $95 \%$ at least once if the relative abundance of this unitag among all busage tags or dt 40 tags is at least $4.55 * 10^{-5}$ or $4.69 * 10^{-5}$ respectively.

Tag to gene assignment using bursal cDNAs, the Ensembl transcript build and the genome sequence

Successful mapping of SAGE tags to reference sequences is influenced by the quality of the sequences, the complexity of the reference sequence datasets and the prevalence of polymorphisms within the tag sequences. It was therefore decided to first search for matches within a bursal cDNA collection which represents the best possible reference dataset, as it was derived from the same tissue and genetic background as the busage library. Subsequently, unitags were mapped to the Ensembl transcript build and finally the chicken genome sequence. Unitags found in a previous dataset were not searched for any more in the next. To

Table I: SAGE and unitag collections

\begin{tabular}{|c|c|c|c|c|}
\hline & SAGE tags & Unitags & $\begin{array}{l}\text { Average frequency of } \\
\text { matching SAGE tags } \\
\text { within library }\end{array}$ & $\begin{array}{c}\text { Average count of SAGE } \\
\text { tag per unitag }\end{array}$ \\
\hline Busage & 65,798 & 24,064 & 2.73 & 4.63 \\
\hline Dt40sage & 63,770 & 21,308 & 2.99 & 4.97 \\
\hline Total & 129,568 & 38,212 & & 3.39 \\
\hline
\end{tabular}


Table 2: Unitag mapping to reference datasets

\begin{tabular}{|c|c|c|c|c|}
\hline & Dataset matches of unitag & Unitags & Candidate tags & Average count of SAGE tags per unitag \\
\hline \multirow[t]{3}{*}{ Bursal cDNA } & & 3,030 & 26,044 & 6.89 \\
\hline & 1 & 2,997 & & 6.90 \\
\hline & $>1$ & 33 & & 5.15 \\
\hline \multirow[t]{3}{*}{ Ensembl transcript build } & & 2,934 & 208,048 & 9.93 \\
\hline & 1 & 2,275 & & 10.60 \\
\hline & $>1$ & 659 & & 7.63 \\
\hline \multirow[t]{3}{*}{ Genome } & & 14,505 & $9,091,924$ & 2.84 \\
\hline & 1 & 13,427 & & 2.83 \\
\hline & $>1$ & 1,078 & & 2.91 \\
\hline Total matching & & 20,469 & & 4.45 \\
\hline Non-matching & & 17,743 & & 2.17 \\
\hline
\end{tabular}

facilitate the searches, candidate tags starting with the CATG tetra-nucleotide were extracted from each reference dataset prior to analysis.

As expected the highest rate of matching to total candidate tags was found for the bursal cDNA collection $(3,030$ of 26,044 candidate tags matched unitags), followed by the Ensembl transcript build $(2,934$ of 208,048$)$ and the genome $(14,505$ of $9,091,924)$ (Table 2$)$. Some unitags mapped more than once within a dataset making an unambiguous assignment difficult. In comparison to the complexity of the dataset, multiple hits occurred more frequently in the bursal cDNA $(33 / 26,044 ; 0.0012 \%)$ and Ensembl dataset $(637 / 208,048,0.0031 \%)$ than in the genome $(1,003 / 9,091,924 ; 0.0001 \%)$. This can be explained if these transcript collections are not completely normalized or if there is bias for certain sequence motifs within gene transcripts. Manual analysis of the bursal cDNAs revealed that most of the multiple unitag matches were due to alternative processing of transcripts originating from the same locus (data not shown). A relatively large fraction of unitags $(17,743 / 38,212 ; 46.4 \%)$ did not match to any reference dataset. It is currently impossible to analyze this in more detail, but sequencing errors, polymorphisms and positions of tags on exon/exon boundaries are likely to explain the missed hits [12]. Nonmatching unitags have a significantly lower average count of SAGE tags than matching unitags (2.1 versus 4.65 ) suggesting that they either over-represent lowly expressed genes or are artifacts of the SAGE technique.

SAGE tags are expected at the position of the NlaIII site closest to the polyA tail of the transcript, but alternative transcript processing as well as incomplete NlaIII digestion or internal priming can produce upstream tags.
Indeed, when the positions of the matching candidate tags were analyzed for bursal cDNA transcripts, about $40 \%$ of the tags matched to non-last positions (data not shown).

\section{Mapping of tags to the genome}

Most interesting from the perspective of gene discovery are the 13,427 unitags without transcript match, but with a single match in the genome (Table 3 ). When the positions of these tags within the genome were correlated with the positions of the Ensembl transcripts, 1,637 fell within annotated transcript boundaries indicating that they are located on missed or incomplete exons. To see whether the remaining tags were located in the neighborhood of already identified transcripts, the numbers of tags falling within regions of defined length upstream and downstream of the Ensembl transcripts were determined. Indeed many tags map very close to annotated transcripts with a strong preference for the region downstream of the transcript, as would be expected, if the tag matches the missed 3' end of an annotated Ensembl gene. Since not all tags are derived from the most 3 ' transcript position, the tags matching immediately upstream of transcripts might indicate missed 5 ' exons. Some of the tags mapped close to upstream and downstream transcripts (12 at the 500 base distance limit), perhaps indicating that these transcripts belong together. At a distance limit of 5000 bases, 7,169 tags mapped into the neighborhood of annotated transcripts; 5,627 downstream, 669 upstream and 1,061 both upstream and downstream. When the distance limit was extended to 10,000 bases, the number of downstream matching tags was only marginally increased to 5,627 whereas the number of dual positioned tags more than doubled to 2,101. This indicates that at distances over 5000 bases the tag assignment to the neighboring tran- 
Table 3: Locations of unitags having a single match in genome but no transcript match

\begin{tabular}{|c|c|c|c|c|c|c|}
\hline & Unitags & $\begin{array}{c}\text { Bases searched } \\
\text { next to } \\
\text { annotated } \\
\text { Ensembl } \\
\text { transcripts }\end{array}$ & Matching unitags & $\begin{array}{c}\text { Matches only } \\
\text { downstream of } \\
\text { Ensembl } \\
\text { transcripts }\end{array}$ & $\begin{array}{c}\text { Matches only } \\
\text { upstream of } \\
\text { Ensembl } \\
\text { transcripts }\end{array}$ & $\begin{array}{c}\text { Matches } \\
\text { upstream and } \\
\text { downstream of } \\
\text { Ensembl } \\
\text { transcripts }\end{array}$ \\
\hline Total & 13,427 & & & & & \\
\hline $\begin{array}{l}\text { Within Ensembl } \\
\text { transcript } \\
\text { boundaries }\end{array}$ & 1,637 & & & & & \\
\hline \multirow{6}{*}{$\begin{array}{l}\text { Outside Ensembl } \\
\text { transcript } \\
\text { boundaries }\end{array}$} & 11,177 & 100 & 409 & 362 & 46 & 1 \\
\hline & & 200 & 732 & 668 & 64 & 2 \\
\hline & & 500 & $\mathrm{I}, 65 \mathrm{I}$ & 1,496 & 143 & 12 \\
\hline & & 1,000 & 2,896 & 2,553 & 262 & 81 \\
\hline & & 5,000 & 7,169 & 5,439 & 669 & $I, 06 \mid$ \\
\hline & & 10,000 & 8,639 & 5,627 & 911 & 2,101 \\
\hline
\end{tabular}

Table 4: Analysis of unitags mapping 5' of or within Ensembl transcript boundaries. \#

\begin{tabular}{|c|c|c|c|c|}
\hline Unitag & EnsembI ID & BLAST result \#\# & $\begin{array}{l}\text { Supporting } \\
\text { bursal EST }\end{array}$ & $\begin{array}{l}\text { Unitag relationship to } \\
\text { Ensembl transcript \#\# }\end{array}$ \\
\hline \multicolumn{5}{|l|}{ Unitags mapping 5' } \\
\hline CATGCTGCTCGCACGAGCCCT & ENSGALT00000002525.I & Q9W7P7 & rikenI_|7|I2r| & Upstream 5' exon \\
\hline CATGGCGGGGTTCCCGGGGCA & ENSGALT00000005092.I & $\begin{array}{l}\text { PEF protein with a long N- } \\
\text { terminal hydrophobic } \\
\text { domain }\end{array}$ & rikenI_I8i20rI & $\begin{array}{l}\text { Upstream } 5 \text { ' exon (EST } \\
\text { supports two additional } 5 \text { ' } \\
\text { exons) }\end{array}$ \\
\hline CATGCTCCTGCTGCTGGCTGG & ENSGALT0000000952I.I & LAC_CHICK & dkfz426_24a5rl & Upstream 5 ' exon \\
\hline CATGAGGCACCTCCTGTTGGC & ENSGALT0000000 I 476.1 & GR78_CHICK & rikenI_25cl4rI & $\begin{array}{l}5^{\prime} \text { upstream/Exon I (EST } \\
\text { supports one additional } 5 \text { ' } \\
\text { exon) }\end{array}$ \\
\hline CATGGCCGCCCAAGGAGAGCC & ENSGALT00000004055.I & RAN_CHICK & riken I_25b20rI & $\begin{array}{l}5 \text { ' upstream/Exon I (EST } \\
\text { supports one additional 5' } \\
\text { exon) }\end{array}$ \\
\hline
\end{tabular}

\section{Unitags mapping within transcript boundaries}

\begin{tabular}{|c|c|c|}
\hline CATGTACTGGTTGTCTGTTTT & ENSGALT00000025884 & HGI4_CHICK \\
\hline CATGCATAGAGGCTTTATTGC & ENSGALT00000021336 & $\begin{array}{l}\text { Aldo-keto reductase family } \\
\text { I member }\end{array}$ \\
\hline CATGTTGGGACTCACCACTCT & ENSGALT00000000504 & No description \\
\hline CATGGTCACCCTAGTAAATAG & ENSGALT00000009677 & Protein kinase $C$, beta type \\
\hline CATGTAAAGTGTTAGCTGTAC & ENSGALT00000006857 & ITF2_CHICK \\
\hline CATGTTACCTGCAACCTGCTG & ENSGALT0000002I577 & Centromeric protein $\mathrm{E}$ \\
\hline CATGGGATATACTGAAAATCT & ENSGALT00000009956 & $\begin{array}{l}\text { T-cell activation leucine } \\
\text { repeat-rich protein }\end{array}$ \\
\hline CATGGGCTGGTTGGTTTTTGT & ENSGALT00000028428 & No description \\
\hline CATGGTCAAGTACAACTCTTA & ENSGALT00000022583 & $\begin{array}{l}\mathrm{Bcl}-2 \text {-associated } \\
\text { transcription factor }\end{array}$ \\
\hline
\end{tabular}

$\begin{array}{ll}\text { dkfz426_I3hI6rl } & \text { Intron 4-5 } \\ d k f z 426 \_3 h \mid 2 r l & \text { Intron 8-9 } \\ \text { dkfz426_|3d22rl } & \text { Intron 5-6/Exon6 } \\ \text { dkfz426_38fl6rl } & \text { Intron 14-15 } \\ \text { dkfz426_|4i24rl } & \text { Intron 8-9 } \\ \text { dkfz426_I7a2Irl } & \text { Intron 28-29 } \\ \text { dkfz426_4Id20rl } & \text { Intron I-2 } \\ \text { dkfz426_43g3rl } & \text { Intron 2-3 } \\ \text { dkfz426_I2n7rl } & \text { Intron 8-9 }\end{array}$

\# Only a few representative examples are shown

\#\# BLAST results are abbreviated

\#\#\#nitag aligns within an intron or exon or lies across an intron/exon or upstream sequence/exon boundary 
scripts is becoming increasingly ambiguous, and the tags might in fact correspond to entirely missed genes.

\section{Relationship of genome mapping unitags to Ensembl transcripts}

To further investigate those unitags mapping close to the 5 ' boundary of Ensembl transcripts or within transcript boundaries to the genome, the bursal EST database [11] was searched for ESTs matching the tags in the sense strand orientation. These ESTs were then aligned to the chicken genome sequence and the neighboring Ensembl gene predictions. As many ESTs linked the SAGE tags to the Ensembl transcripts, this provided independent experimental evidence that these tags are indeed derived from non-annotated parts of these transcripts (Table 4).

To confirm that the distribution of the tags downstream of Ensembl transcripts is statistically significant, their posi- tions were compared to the positions of simulated tags generated by randomly selecting 21 bp sequences in the genome beginning with the 'CATG' tetra-nucleotide. This comparison shows that the real tags map closer to the 3' end of the Ensembl predicted coding sequences (CDS) than the simulated tags providing strong evidence that most of the closely positioned tags are indeed related to the predicted transcripts (Figure 2).

If one summarizes the unitag to transcript mappings, 5,964 unitags map directly to transcripts, 1,637 map to not annotated sequences within the limit of the Ensembl transcripts and 7,169 map to within 5,000 bases of annotated transcript boundaries (Table 5). This leaves about $20 \%(4,621$ out of 19,391 total) bona fide unitags unaccounted for which might be taken as an estimate for the percentage of genes present in the released genome

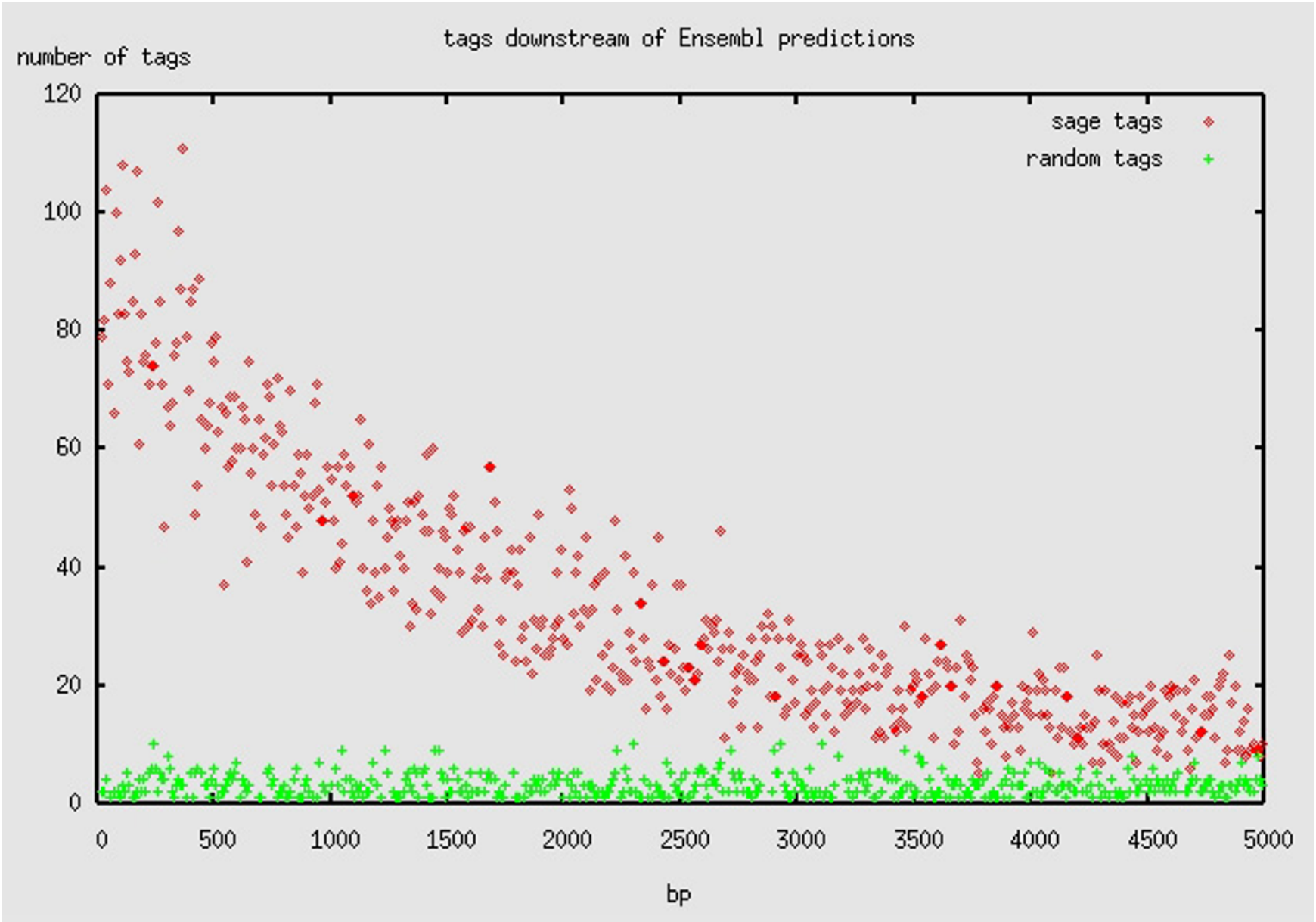

Figure 2

Mappings of SAGE unitags downstream of Ensembl transcripts compared to simulated genomic tags. The number of tags falling within windows of $10 \mathrm{bp}$ is plotted on the $y$-axis whereas the distance from the 3 ' end of the nearest predicted Ensembl transcript is plotted on the $\mathbf{x}$-axis. Sage unitags coordinates are indicated by crosses and randomly selected tag coordinates by diamonds. 
Table 5: Unitag mapping to transcripts

\begin{tabular}{|c|c|c|c|c|c|}
\hline & Unitag & $\begin{array}{c}\text { Match to annotated } \\
\text { transcript }\end{array}$ & $\begin{array}{l}\text { Match to genome } \\
\text { within boundaries of } \\
\text { annotated transcript }\end{array}$ & $\begin{array}{c}\text { Match next to } \\
\text { annotated transcript } \\
\text { using } 5000 \text { base cut- } \\
\text { off }\end{array}$ & $\begin{array}{l}\text { Match distant from } \\
\text { annotated transcript }\end{array}$ \\
\hline Total & 38,212 & & & & \\
\hline Without match & 17,743 & & & & \\
\hline $\begin{array}{l}\text { With only multiple } \\
\text { genome matches }\end{array}$ & 1,078 & & & & \\
\hline $\begin{array}{l}\text { With match to } \\
\text { annotated transcripts } \\
\text { or single genome } \\
\text { match }\end{array}$ & 19,391 & 5,964 & 1,637 & 7,169 & 4,621 \\
\hline
\end{tabular}

sequence, but absent from the Ensembl transcript collection. Nevertheless, one needs to bear in mind that this calculation includes a number of uncertainties. It is for example possible that the 5000 base limit is too large, since only $5 \%$ of 3 ' UTR sequences in the human transcriptome are reported to be over 2,000 bps according to NCBI's AceView database http://www.ncbi.nih.gov/IEB/ Research/Acembly/ or that both SAGE and the gene predictions have missed a substantial number of lowly expressed transcripts. In these cases, the estimate of the percentages of missed genes would increase.

Significant gene expression differences between bursal cells and the DT40

One of the goals of this SAGE analysis was the identification of differentially expressed transcripts between the two libraries and the significance of count differences for the busage and the dt40sage tags were calculated for each unitag. In total 629 unitags showed p values below 0.01 suggesting that the corresponding transcripts are differentially expressed in bursal cells and DT40. In contrast to this, the false discovery rate (FDR) controlling procedure of Benjamin \& Hochberg would admit the first 229 genes at an FDR of 5\% [13]. Twenty-five of the most significant unitags mapping to bursal cDNAs are listed in Table 6.

To verify the validity of the SAGE data, semi-quantitative PCR was performed using primers close to the tags for 27 transcripts (Figure 3). This confirmed the expression pattern suggested by SAGE tag counts in the majority (21 out of 27) of the cases. Certainly, these PCR results could not be explained by the statistical variation in the SAGE data alone (FDR below 5\% vs. FDR of 22\% indicated by PCR). Although more analysis is needed to find out which differ- entially expressed genes are related to differences in the behavior of bursal B cells and DT40, the freely available SAGE repository will be a good resource to select candidates for more detailed investigations.

\section{Conclusions}

The mapping of the SAGE tags to the recently released cDNA collections and the chicken genome has been useful to assess the completeness and accuracy of the current transcript catalog. On the positive side, it appears that the transcript build may have missed only a low percentage of genes, since relatively few tags map to genome regions far away from annotated transcription units. On the downside, fewer than 6,000 of over 19,000 tags with matches to reference sequences could be mapped to transcripts. The majority of the tags missed in transcripts are positioned downstream of annotated transcripts with a minority mapping upstream or within the genomic boundaries of transcripts. The most straightforward explanation for this is that many transcripts in the current version of the chicken transcriptome do not accurately reflect the $3^{\prime}$ and the 5 ' ends of transcripts. This proposition is independently supported by the comparisons of the bursal full length cDNAs to the Ensembl transcript build which detected discrepancies to Ensembl annotated transcripts for approximately $50 \%$ of the cDNAs [10]. Another explanation for at least part of the missing transcript matches is variability in poly-adenylation and splicing, which seems to account for substantial variety in the human transcriptome [12]. 
Accurate definitions of the transcribed parts of the chicken

Table 6: List of genes differentially expressed in bursal cells and DT40

\begin{tabular}{|c|c|c|c|c|c|}
\hline Unitag & Busage & DT40sage & Significance & Sequence ID\# & Best BLAST result \\
\hline CATGGCAGGGGGCGGAAACCT & 4 & 45 & $2.83 \mathrm{E}-10$ & rikenI_2o24 & (AAH6I765) Hypothetical protein \\
\hline CATGGTGAGCCAAGGTGTTGT & 24 & 82 & $2.06 \mathrm{E}-9$ & riken I_4ml & $\begin{array}{l}\text { (AAH69219) Cold inducible RNA- } \\
\text { binding protein }\end{array}$ \\
\hline CATGCAGAAATAAGCTTCTCC & 45 & 109 & 4.09E-8 & riken I_7bI5 & $\begin{array}{l}\text { (Q7ZUR6) Similar to muscle-specific } \\
\text { beta I integrin binding protein }\end{array}$ \\
\hline CATGAGCGGGGGCAGCACTTG & 118 & 203 & $5.75 \mathrm{E}-7$ & rikenI_25p23 & (Q90YW7) Ribosomal protein L4 \\
\hline CATGCTGGAAGAAAGAATAAC & 46 & 114 & $1.92 \mathrm{E}-8$ & riken I_32cll & $\begin{array}{l}\text { (Q9YGQ I) Peptide elongation factor } \\
\text { I-beta }\end{array}$ \\
\hline CATGCGCTCTCCTTTTAAAAG & 9 & 41 & $2.67 \mathrm{E}-6$ & rikenI_I513 & $\begin{array}{l}\text { (CAA3 I409) Chinese hamster } \\
\text { asparagine synthetase }\end{array}$ \\
\hline CATGGATGGCCAGCAAGTGTT & 29 & 4 & I.I7E-5 & riken I_4k19 & $\begin{array}{l}\text { (PI3796) L-plastin (Lymphocyte } \\
\text { cytosolic protein I) }\end{array}$ \\
\hline CATGTCCGTGGCATCCTTTGA & 0 & 16 & $1.18 \mathrm{E}-5$ & riken I_24e23 & $\begin{array}{l}\text { (Q8BGQ8) Heterogeneous nuclear } \\
\text { ribonucleoprotein K }\end{array}$ \\
\hline CATGGCTTTGGAATATTTGAC & 25 & 3 & $2.90 \mathrm{E}-5$ & riken I_2f9 & $\begin{array}{l}\text { (AAH46I52) Selenoprotein P } \\
\text { precursor }\end{array}$ \\
\hline CATGGAGTCCATAACACGGCG & 21 & 2 & $6.88 \mathrm{E}-5$ & riken I_34m 12 & $\begin{array}{l}\text { (Q96CJI) Testosterone regulated } \\
\text { apoptosis inducer and tumor } \\
\text { suppressor }\end{array}$ \\
\hline CATGCAAAGTGCCCTTGGCTT & 17 & 1 & I.46E-4 & riken I_IOgI9 & (P3028I) GI/S-specific cyclin D3 \\
\hline CATGTAAGCCAATTCTGAACC & 19 & 1 & 4.09E-5 & rikenI_33al8 & $\begin{array}{l}\text { (Q8JHJ4) TNF family B cell activation } \\
\text { factor }\end{array}$ \\
\hline CATGTTGTACACACGGGCACT & 11 & 0 & $5.79 \mathrm{E}-4$ & riken I_5gl2 & (Q90YB0) FEN-I nuclease \\
\hline CATGTGCCCGTGACCCCCATC & 2 & 16 & $6.12 \mathrm{E}-4$ & rikenI_4nI5 & $\begin{array}{l}\text { (Q13200) } 26 \text { S proteasome non- } \\
\text { ATPase regulatory subunit } 2\end{array}$ \\
\hline CATGTCGTGCTCTGTGCCTCC & 5 & 26 & $9.28 \mathrm{E}-5$ & riken I_2i9 & (Q90W60) XNop56 protein \\
\hline CATGCTTTCTGCTTTGACTTT & 21 & 4 & $9.42 \mathrm{E}-4$ & rikenI_I2pI6 & $\begin{array}{l}\text { (P22794) Ecotropic viral integration } \\
\text { site } 2 A \text { protein }\end{array}$ \\
\hline CATGTTTGTGCATAGCTGTCC & 5 & 28 & I.I7E-5 & rikenI_30e3 & $\begin{array}{l}(\mathrm{Q} 9 \mathrm{IXC} 8) \text { Similar to death-associated } \\
\text { protein }\end{array}$ \\
\hline CATGGCCGGGCGCCCCACCAG & 0 & 15 & $2.4 I E-5$ & rikenI_I5il3 & (Q99P44) Leucine aminopeptidase \\
\hline CATGGGACCAACAAATAAAGC & 19 & 4 & 0.0027 & rikenI_4ol0 & $\begin{array}{l}\text { (P97440) Histone RNA hairpin- } \\
\text { binding protein }\end{array}$ \\
\hline CATGAAAATGTACTGTGCTAA & 2 & 13 & 0.0036 & riken I_20p3 & $\begin{array}{l}\text { (P34022) Ran-specific GTPase- } \\
\text { activating protein }\end{array}$ \\
\hline CATGTATACAGAACTGCTGGA & 8 & 0 & 0.0044 & rikenI_2i24 & $\begin{array}{l}\text { (Q9UMR2) ATP-dependent RNA } \\
\text { helicase DDX19 }\end{array}$ \\
\hline CATGGCCAAATTAGAGGAGTG & I & 10 & 0.0051 & riken I_32cll & $\begin{array}{l}\text { (Q9YGQI) Peptide elongation factor } \\
\text { I-beta }\end{array}$ \\
\hline CATGCTACGCTGTGTCTGCCA & 11 & 1 & 0.0062 & rikenI_2m I4 & $\begin{array}{l}\text { (AAQ20009) Heterogeneous nuclear } \\
\text { ribonucleoprotein HI-like protein }\end{array}$ \\
\hline CATGCTCTCCGGTGGTACAAT & 0 & 7 & 0.0070 & riken I_32cll & $\begin{array}{l}\text { (Q9YGQ I) Peptide elongation factor } \\
\text { I-beta }\end{array}$ \\
\hline CATGTTGATTCCTATGCTAAA & 7 & 0 & 0.0087 & rikenI_3a6 & $\begin{array}{l}\text { (Q9HI65) B-cell lymphoma/leukemia } \\
\text { IIA }\end{array}$ \\
\hline
\end{tabular}

\# only unitags matching bursal cDNAs are listed \#\# BLAST results are abbreviated

genome is highly desirable not only to ascertain the correct ORFs, but also to identify transcription and translational control sequences often located in $5^{\prime}$ and $3^{\prime}$ untranslated regions. It should be interesting to use the genomic positions of the missed transcript tags in combination with current gene finder algorithms to improve transcript coverage. Many of the missed tags are close to already annotated exons facilitating this task. It should also be possible to use promising tag sequences to screen cDNA libraries for clones whose sequence will identify missed genes or exons. The riken 1 bursal cDNA library is of excellent quality and should be suitable for this purpose. 


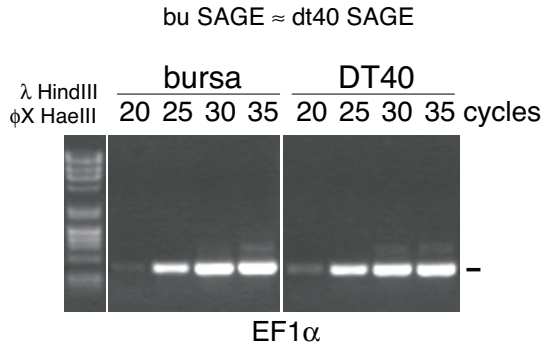

(431, 352; 1.83E-02)

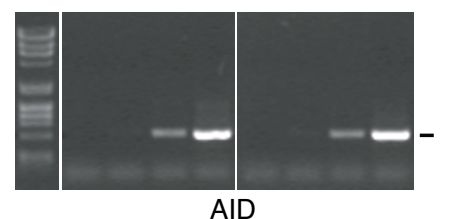

$(2,2 ; 9.33 \mathrm{E}-01)$

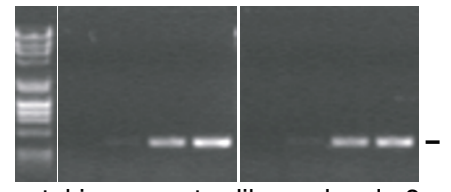

cytokine receptor-like molecule 9 $(19,19 ; 8.72 \mathrm{E}-01)$

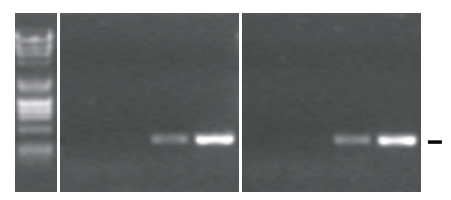

putative growth hormone-like protein-1 (16, 10; 3.28E-01)

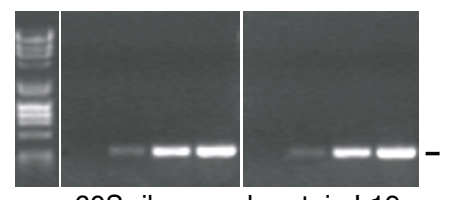

60S ribosomal protein L19 $(43,25 ; 5.15 \mathrm{E}-02)$ bu SAGE > dt40 SAGE

bursa DT40

2025303520253035 cycles

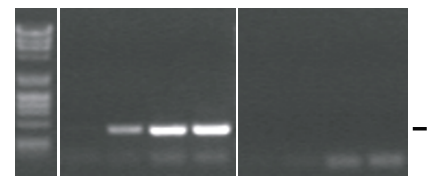

ovocalyxin-32

$(118,1 ; 1.14 \mathrm{E}-19)$

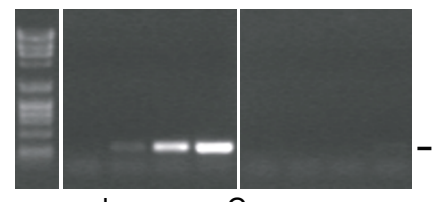

lysozyme $\mathrm{C}$ precursor

$(127,2 ; 1.61 \mathrm{E}-18)$

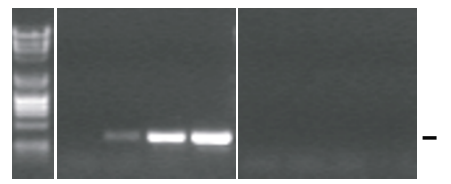

prostaglandin D2 synthase

$(56,1 ; 1.11 \mathrm{E}+01)$

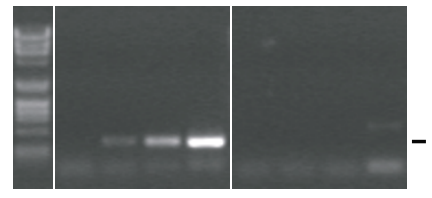

ChEST952g8

$(1179,22 ; 0.0)$

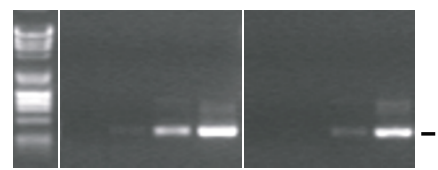

cathepsin $\mathrm{S}$ preproprotein precursor $(18,1 ; 7.74 \mathrm{E}+09)$ bu SAGE $<$ dt40 SAGE

bursa DT40

2025303520253035 cycles

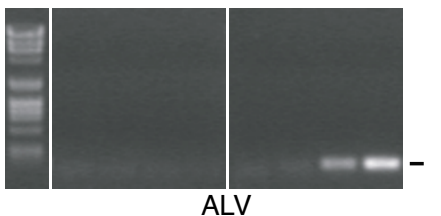

$(3,90 ; 5.19 \mathrm{E}-08)$

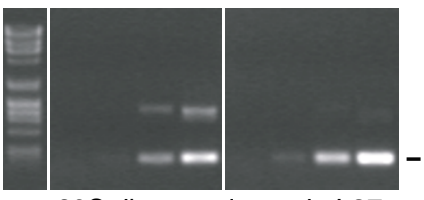

$60 S$ ribosomal protein $\mathrm{L} 37 \mathrm{a}$

$(6,79 ; 6.74 \mathrm{E}-04)$

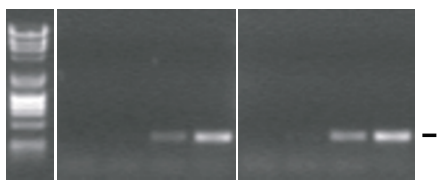

PGAM1

$(15,116 ; 2.23 E-05)$

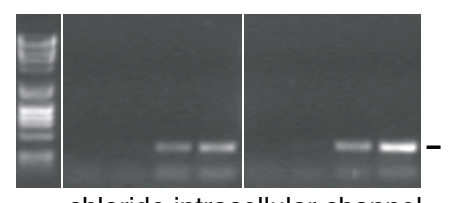

chloride intracellular channel protein $2(5,32 ; 3.26 \mathrm{E}+08)$

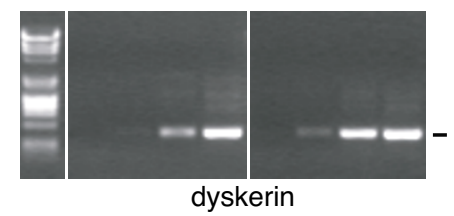

$(6,30 ; 3.33 \mathrm{E}+08)$

\section{Figure 3}

Confirmation of differential gene expression using semi-quantitative PCR. Primers derived from reference genes for SAGE tags were used for the amplification of cDNA from bursal cells and DT40 employing different cycle numbers as indicated on top of the lanes. Based on the SAGE tag counts, the reference genes were classified as likely to be equally expressed (left part), higher expressed in bursal cells (middle part) or higher expressed in DT40 (right part). The size of the expected PCR product is indicated by a bar adjacent to the gel image. The numbers of tags found for the busage and dt40sage libraries as well as the calculated significance for differential expression are indicated in brackets under the gene names. 
Although the presented SAGE data provides valuable information about the expression levels of many genes in bursal cells and the DT40 cell line, the full potential of SAGE for gene expression profiling could not be exploited due to the difficulties in tag to gene assignment. Nevertheless, this first SAGE analysis in the chicken lays the basis for further studies. SAGE has the advantage that data from different experiments and laboratories are easily comparable as the tag sequences serve as a common standard. Accumulation of additional data will increasingly facilitate the interpretation of results because bona fide tags will be distinguished from artifacts by being replicated and even polymorphic tags will eventually be defined and assigned to their corresponding transcripts.

\section{Methods \\ LongSAGE library construction}

Total RNA from bursal tissue of chicken 20 day old CBinbred chicks and from DT40 Cre1 cells [14] was extracted using TRIzol reagent (Invitrogen) according to the manufacturer's instructions. PolyA RNA was isolated using the mRNA DIRECT kit from Dynal http://www.dynal.no. The RNA bound to oligo(dT) ${ }_{25}$ magnetic beads was immediately used for the construction of a LongSAGE library $[1,3]$ following a modified protocol as described previously [15]. High fidelity PfuUltra (Stratagene) polymerase was used for the PCR amplification step. The SAGE libraries from bursal tissue and DT40 were named busage and dt40sage respectively. For each library, distinct Linker/ Primer combinations were used to exclude accidental amplification of ditags from the other library.

\section{Sequencing of SAGE library clone inserts}

The pZero-1 (Invitrogen) plasmids containing SAGE ditags as multimeric inserts were transformed into E. coli. Zeocin resistant colonies transformed by the plasmids were grown at low density on agar plates, picked and directly suspended in 50 microliters of $\mathrm{H}_{2} \mathrm{O}$. This suspension was heated at $95^{\circ} \mathrm{C}$ for 10 minutes and stored at $20^{\circ} \mathrm{C}$ until further processing. The PCR amplification used primers from the plasmid backbone, M13 forward and reverse. Sequencing was performed using the Big Dye v3.1 ready reaction mix (Applied Biosystems) and a nested primer (SSP2) from the plasmid poly-linker. Reactions were analyzed on an ABI 3730 DNA Analyzer (Applied Biosystems). The raw sequencing files were processed as described previously [16].

\section{Ditag, tag and unitag definition}

The library insert sequences were searched for ditags in which the flanking CATG tetra-nucleotides are separated by a spacer sequence of more than 31 and less than 37 bases. Ditags of identical sequence were entered only once for each library to avoid the possibility of entering PCR amplification artifacts. The ditags were then divided into two SAGE tags of 21 bases including the CATG tetranucleotides. The combined SAGE tag collections of both libraries were normalized to generate a collection of unitags possessing unique tag sequences. A low number of tags ( 197 of 129,568 total tags) were found to be identical to the sequences of the linker tags used for the library construction and therefore were removed. Care was taken to minimize the possibility of tag sequence errors by using a high fidelity polymerase for the PCR amplification step of the library construction and by rejecting any ditag sequences which contained even a single ambiguous base call or a PHRED score lower than 10. It is possible that some unitags are due to sequencing errors, but these artificial tags are unlikely to match transcript or genome sequences.

\section{Tag-to-gene mapping}

To map the unitags to reference sequences, candidate tags were extracted from i) full length bursal cDNA sequences [10], ii) the Ensembl transcript build $\mathrm{ftp}: / /$ ftp.ensembl.org/pub/current chicken/data/fasta/cdna/

and iii) the chicken chromosome sequences $\mathrm{ftp}: / /$ ftp.ensembl.org/pub/current chicken/data/fasta/dna/.

Candidate tags in the transcript datasets were extracted only in the sense orientation whereas both strands of the chromosome sequences were searched. The SAGE tags, unitags and candidate tags together with relevant information concerning their positions and frequencies were entered into tables of a relational database to facilitate further analysis.

Unitag matches were sequentially searched for in the bursal cDNA collection, the Ensembl transcript build and the Genome. Once a match had been identified, that tag fell out of the remaining search process and only matches of identical sequences were accepted. To relate the position of matching unitags in the genome sequence to the Ensembl transcripts, the chromosome coordinates of the Ensembl transcripts and their orientation were extracted from their headers. The database table structure, all tabulated entries as well as the FOUNTAIN software [17] used for the analysis is freely available for download under http://pheasant.gsf.de/SAGE/download/ and http:// pheasant.gsf.de/DEPARTMENT/FOUNTAIN.html.

\section{Calculation of the significance of SAGE count differences}

To evaluate the significance of SAGE tag count differences between the libraries for each unitag, we used Fishers exact test [18] since it is most easy to use, has exact size and does not require specifying hyper-parameters like for a Bayesian approach. As usual, no method to account for multiple testing was used, so p-values were just used as a convenient tool to rank the unitags. 
Isolation of mRNA from bursal cells and DT40

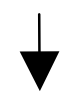

SAGE library construction using the LongSAGE 21 protocol

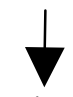

Clonal PCR amplification of plasmid SAGE tag inserts

\section{SAGE library construction}

\section{Generation of SAGE \\ tag databases}

Tag storage and update of the unitag table

129,568 tags and 38,212 unitags defined

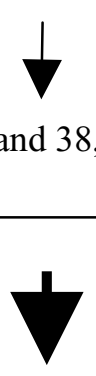

Single pass sequencing of the PCR templates

Ditag resolution and discarding of duplicates

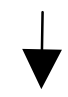

Search for unitag matches in bursal cDNAs

Search for matches in the chicken unigene collection

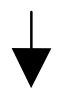

Search for matches in the chicken genome sequence

\section{Tag to Gene assignment}

\section{Figure I}

Outline of SAGE tag production and reference gene assignment. 


\section{Semi-quantitative PCR}

cDNA was synthesized from bursal tissue and DT40 Cre1 cell line using the SuperScript Preamplification System (Invitrogen). Primers were designed to amplify a region of a few hundred base pairs encompassing the SAGE unitag sequence of the reference transcript. PCR amplification was performed using the Expand Long Template PCR System (Roche) under the following conditions: $2 \mathrm{~min}$ initial incubation at $93^{\circ} \mathrm{C} ; 20,25,30$ and 35 cycles consisting of $10 \mathrm{sec}$ at $93^{\circ} \mathrm{C}, 30 \mathrm{sec}$ at $65^{\circ} \mathrm{C}$ and $5 \mathrm{~min}$ at $68^{\circ} \mathrm{C}$ with $20 \mathrm{sec}$ elongation per cycle.

\section{Authors' contributions}

$\mathrm{JMB}$ and $\mathrm{MBW}$ conceived the project. MBW, $\mathrm{RBC}, \mathrm{HA}$ and JMB participated in the design of the study and its coordination. MBW constructed the libraries. MBW, RBC, NH, $\mathrm{CJ}, \mathrm{MS}, \mathrm{MC}$ and YDW performed clone management, sequencing and data analysis. HA performed confirmation PCR analysis. MBW, RBC, AMK, EE, VL and JMB performed bioinformatics and statistical analysis. AMK and JMB programmed the FOUNTAIN software package to include the SAGE analysis modules. MBW, RBC, AMK, HA, VL and JMB helped draft the manuscript. All authors read and approved the final manuscript.

\section{Acknowledgments}

This work was supported by the EU grants 'Genetics in a cell line' and 'Mechanisms of gene integration'. We would like to thank Kenji Imai for stimulating discussion.

\section{References}

I. Velculescu VE, Zhang L, Vogelstein B, Kinzler KW: Serial analysis of gene expression. Science 1995, 270:484-487.

2. Madden SL, Wang CJ, Landes G: Serial analysis of gene expression: from gene discovery to target identification. Drug Discov Today 2000, 5:4I 5-425.

3. Saha S, Sparks AB, Rago C, Akmaev V, Wang CJ, Vogelstein B, Kinzler $\mathrm{KW}$, Velculescu VE: Using the transcriptome to annotate the genome. Nat Biotechnol 2002, 20:508-5I 2 .

4. Modrek B, Lee C: A genomic view of alternative splicing. Nat Genet 2002, 30:13-19.

5. Pike KA, Baig E, Ratcliffe MJ: The avian B-cell receptor complex: distinct roles of Igalpha and Igbeta in B-cell development. Immunol Rev 2004, 197:10-25.

6. Hayward WS, Neel BG, Astrin SM: Activation of a cellular onc gene by promoter insertion in ALV-induced lymphoid leukosis. Nature 198I, 290:475-480.

7. Neiman PE, Clurman BE, Lobanenkov VV: Molecular pathogenesis of myc-initiated B-cell lymphomas in the bursa of Fabricius. Curr Top Microbiol Immunol 1997, 224:23 I-238.

8. Neiman PE, Ruddell A, Jasoni C, Loring G, Thomas SJ, Brandvold KA, Lee Rm, Burnside J, Delrow J: Analysis of gene expression during myc oncogene-induced lymphomagenesis in the bursa of Fabricius. Proc Natl Acad Sci USA 2001, 98:6378-6383.

9. International Chicken Genome Sequencing Consortium: Sequence and comparative analysis of the chicken genome provide unique perspectives on vertebrate evolution. Nature 2004, 432:695-7I6.

10. Caldwell R, Kierzek A, Arakawa H, Bezzubov Y, Zaim J, Fiedler P, Kutter S, Blagodatski A, Kostavska D, Koter M, Carninci P, Hayashizaki $Y$, Buerstedde JM: Full-length cDNAs from bursal lymphocytes to facilitate gene function analysis. Genome Biology in press.

II. Buerstedde JM, Arakawa H, Watahiki A, Carninci PP, Hayashizaki YY, Korn B, Plachy J: The DT40 website: Sampling and connecting the genes of a B cell line. Nucl Acid Res 2002, 30:230-23I.
12. Pleasance ED, Marra MA, Jones SJ: Assessment of SAGE in transcript identification. Genome Res 2003, I3:I203-I2I5.

13. Benjamini Y, Hochberg Y: Controlling the False Discovery Rate - A Practical and Powerful Approach to Multiple Testing. J Roy Stat Soc B Met 1995, 57(I):289-300.

14. Arakawa $H$, Lodygin D, Buerstedde JM: Mutant loxP vectors for selectable marker recycle and conditional knock-outs. $B M C$ Biotechnol 200I, I:7.

15. Wahl M, Shukunami C, Heinzmann U, Hamajima K, Hiraki Y, Imai K: Transcriptome analysis of early chondrogenesis in ATDC5 cells induced by bone morphogenetic protein 4. Genomics 2004, 83:45-58

16. Abdrakhmanov I, Lodygin D, Geroth P, Arakawa H, Law A, Plachy J, Korn B, Buerstedde JM: A large database of chicken bursal ESTs as a resource for the analysis of vertebrate gene function. Genome Res 2000, 10:2062-2069.

17. Buerstedde JM, Prill F: FOUNTAIN: a JAVA open-source package to assist large sequencing projects. BMC Bioinformatics 200 I, 2:6.

18. Ruijter JM, Van Kampen AH, Baas F: Statistical evaluation of SAGE libraries: consequences for experimental design. Physiol Genomics 2002, I I :37-44.

Publish with Bio Med Central and every scientist can read your work free of charge

"BioMed Central will be the most significant development for disseminating the results of biomedical research in our lifetime. "

Sir Paul Nurse, Cancer Research UK

Your research papers will be:

- available free of charge to the entire biomedical community

- peer reviewed and published immediately upon acceptance

- cited in PubMed and archived on PubMed Central

- yours - you keep the copyright
BioMedcentral 